V D. NGUYEN

KODAI MATH. J.

22 (1999), 46-55

\title{
BRAID MONODROMY OF COMPLEX LINE ARRANGEMENTS
}

\author{
Dedicated to the memory of Professor N. Sasakura
}

\author{
NGUYEN VIET DUNG ${ }^{\dagger}$
}

\begin{abstract}
Let $V$ be the complex vector space $C^{l}, \mathscr{A}$ an arrangement in $V$, 1.e. a finite family of hyperplanes in $V$ In [11], Moishezon associated to any algebraic plane curve $\mathscr{C}$ of degree $n$ a braid monodromy homomorphism $\theta \quad F_{s} \rightarrow B(n)$, where $F_{s}$ is a free group, $B(n)$ is the Artin braid group. In this paper, we will determine the braid monodromy for the case when $\mathscr{C}$ is an arrangement $\mathscr{A}$ of complex lines in $C^{2}$, using the notion of labyrinth of an arrangement. As a corollary we get the braid monodromy presentation for the fundamental group of the complement to the arrangement.
\end{abstract}

\section{Introduction}

Let $\mathscr{C}=\{f(x, y)=0\} \in C^{2}$ be a plane algebraic curve. From the 1930's, it is well known (see [9], [17]) that the fundamental group of the complement to $\mathscr{C}, \pi_{1}\left(\boldsymbol{C}^{2} \backslash \mathscr{C}\right)$, can be computed using the van Kampen's method. In [11], Moishezon introduced the notion of braid monodromy of $\mathscr{C}$. Suppose that the projection on the $x$-axis, $p r_{1}: C^{2} \rightarrow C^{1}$, is generic with respect to the curve $\mathscr{C}$. Let $S(\mathscr{C})=\{\alpha \in \mathscr{C} ; \partial f(\alpha) / \partial y=0\}$ and $D(\mathscr{C})$ its image under $p r_{1}$. Then the braid monodromy of $\mathscr{C}$ is a homeomorphism $\theta: \pi_{1}\left(C^{1} \backslash D(\mathscr{C})\right) \rightarrow$ $B\left[p_{1}^{-1}\left(x_{0}\right), \operatorname{pr}_{1}^{-1}\left(x_{0}\right) \cap \mathscr{C}\right]$, where $x_{0} \in C^{1} \backslash D(\mathscr{C})$ is a base point.

An arrangement $\mathscr{A}$ is a finite family of hyperplanes in $C^{l}$. Given an arrangement $\mathscr{A}$, an algorithm to compute the fundamental group of the complement, $\pi_{1}\left(C^{l} \backslash \bigcup_{H \in \mathscr{A}} H\right)$, was proved in [14] when $\mathscr{A}$ is the complexification of a real arrangement. Similar results were obtained in [5] and [16] by different methods. For an arbitrary complex arrangement a standard argument using the Zariski hyperplane section theorem (see e.g. [7]) reduces the problem to the case when $\mathscr{A}$ is an arrangement of complex lines in $\boldsymbol{C}^{2}$. Arvola [1] found an

1991 Mathematics Subject Classification: 14H30, 32S50.

Keywords: hyperplane arrangement, labyrınth, braid monodromy, fundamental group.

${ }^{\dagger}$ The author was supported partially by a TMU research grant and by the National Basic Research Program in Natural Sciences of Vietnam

Received March 30, 1998. 
algorithm to compute the fundamental group of its complement, using an admissible 2-graph, defined by himself. In [6] we suggest another method to compute this fundamental group. Our method based on a construction called labyrinth, which dues to Rudolph [15]. We found that the labyrinth is still useful to study the braid monodromy of an arrangement $\mathscr{A}$ of complex lines in $C^{2}$. In this paper we will show how the braid monodromy of the arrangement $\mathscr{A}$ can be obtained from its labyrinth. Note that the braid monodromy gives also a presentation for the fundamental group of the complement of $\mathscr{A}$. Combining this with a result of Libgober [10], we prove in the corollary 4.6 that the labyrinth of an arrangement $\mathscr{A}$ in $C^{2}$ determines the homotopy type of its complement.

The braid monodromy of a complexified real arrangement was determined by Salvetti [16], Hironaka [8] and Cordovil and Fadacha [4]. Generalizing the notion of admissible graph and the algorithm of Arvola, recently, Cohen and Suciu [3] suggest an algorithm to determine the braid monodromy of an arrangement of complex lines using braided wiring diagram. However, the method we present here is quite different. Moreover, our method gives a concrete algorithm to determine precisely any braid occurring in the conjugation of braid monodromy generators (see the Remark 4.3).

Acknowledgement. The author would like to thank Ha Huy Vui for proposing the use of labyrinth in the arrangement context and Mutsuo Oka for the hospitality at the Department of Mathematics, Tokyo Metropolitan University as well as for many useful discussions.

\section{Braid monodromy}

In this section we recall briefly the notion of the braid monodromy of a plane curve after B. Moishezon [11].

Let $\mathscr{C}=\{f(x, y)=0\} \in C^{2}$ be a plane curve. Suppose that the projection $p r_{1}: C^{2} \rightarrow C^{1}$ onto the $x$-axis is generic with respect to the curve $\mathscr{C}$. Denote by $S(\mathscr{C})$ the set $\{\alpha \in \mathscr{C} ; \partial f(\alpha) / \partial y=0\}$ and $D(\mathscr{C})$ the image of $S(\mathscr{C})$ under the projection $p r_{1}$. For a point $\tilde{x}$ of the $x$-plane $C^{1}$ let $C_{\tilde{x}}$ denote the fiber of the projection $p r_{1}$ over the point $\tilde{x}, C_{\tilde{x}}=\left\{(x, y) \in C^{2} ; x=\tilde{x}\right\}$. For a path $\gamma: I \rightarrow$ $C^{1} \backslash D(\mathscr{C})$, we see easily that the pull-backs $\gamma^{*}\left(p r_{1}\right)$ and $\gamma^{*}\left(\left.p r_{1}\right|_{\mathscr{C}}\right)$ are trivial bundles. We have then the homeomorphisms

$$
\left(p r_{1}^{-1}(\gamma(0)), p r_{1}^{-1}(\gamma(0)) \cap \mathscr{C}\right) \rightarrow\left(p r_{1}^{-1}(\gamma(t)), p r_{1}^{-1}(\gamma(t)) \cap \mathscr{C}\right),
$$

$t \in[0,1]$, induced naturally by a given trivialization of $\left(\gamma^{*}\left(p r_{1}\right), \gamma^{*}\left(\left.p r_{1}\right|_{\mathscr{C}}\right)\right)$. We call this homeomorphism the braid homeomorphism defined over the path $\gamma$, or simply the braid defined over $\gamma$. Let fix a base point $x_{0}$ of the $x$-axis, $x_{0} \in C^{\mathbb{l}} \backslash D(\mathscr{C})$. When $\gamma$ is a loop beginning and ending at $x_{0}$, we obtain a homeomorphism

$$
\left(\boldsymbol{C}_{x_{0}}, \boldsymbol{C}_{x_{0}} \cap \mathscr{C}\right) \rightarrow\left(\boldsymbol{C}_{x_{0}}, \boldsymbol{C}_{x_{0}} \cap \mathscr{C}\right)
$$


This defines a homomorphism

$$
\theta: \pi_{1}\left(C^{1} \backslash D(\mathscr{C}) ; x_{0}\right) \rightarrow B\left[C_{x_{0}}, C_{x_{0}} \cap \mathscr{C}\right],
$$

which is called the braid monodromy of the curve $\mathscr{C}$. Here by $B[P, K]$ we mean the group of isotopy classes of compact support homeomorphisms of a 2-plane $P$ which preserves a fixed finite subset $K \subset P$.

The determination of the braid monodromy is usually carried out in two steps. First, for a point $x_{k} \in D(\mathscr{C})$ we denote by $D_{x_{k}}^{\varepsilon}$ a small disk of radius $\varepsilon$, centered at $x_{k}$. Let fix a point $x_{k}^{\varepsilon}$ on the boundary $\partial D_{x_{k}}^{\varepsilon}$ of this disk and $C_{x_{k}^{\varepsilon}}$ the fiber over this point $x_{k}^{\varepsilon}$. By moving this fiber $C_{x_{k}^{\varepsilon}}$ counterclockwise along the boundary of the disk $D_{x_{k}}^{\varepsilon}$ we obtain a homeomorphism of $C_{x_{k}^{\varepsilon}}$ into itself, preserving $\boldsymbol{C}_{x_{k}^{\varepsilon}} \cap \mathscr{C}$. I gives rise an element of the braid group $B\left[\boldsymbol{C}_{x_{k}^{\varepsilon}}, \boldsymbol{C}_{x_{k}^{\varepsilon}} \cap \mathscr{C}\right]$ and will be called the local braid monodromy of $\mathscr{C}$ at $x_{k}$.

Next, suppose that $D(\mathscr{C})=\left\{x_{1}, \ldots, x_{N}\right\}$. Let $\Gamma_{1}, \ldots, \Gamma_{N}$ be a system of simple paths in $C^{1} \backslash D(\mathscr{C})$ satisfying

1) $\Gamma_{l} \cap \Gamma_{J}=x_{0}, 1 \leq i<j \leq N$.

2) Each $\Gamma_{l}$ connects $x_{0}$ with $x_{i}^{\varepsilon}$ and $\Gamma_{l} \cap D(\mathscr{C})=\emptyset$.

Denote by $\gamma_{i}$ the element of $\pi_{1}\left(C^{1} \backslash D(\mathscr{C})\right)$, represented by $\Gamma_{l}, \partial D_{x_{i}}^{\varepsilon} \cdot \Gamma_{l}^{-1}$. The set of all those $\gamma_{i}$ 's is called a good ordered system of generators of $\pi_{1}\left(C^{1} \backslash D(\mathscr{C})\right)$. To find the braid monodromy $\theta$ it suffices to find all $\theta\left(\gamma_{i}\right), 1 \leq$ $i \leq N$. Let $\theta\left(\Gamma_{l}\right)$ be the braid homeomorphism defined over the path $\Gamma_{l}$. Then it is clear that $\theta\left(\gamma_{i}\right)$ is completely determined by the local braid monodromy at $x_{l}$ and the braid $\theta\left(\Gamma_{t}\right)$.

In this paper we will deal with the case when $\mathscr{C}$ is an arrangement $\mathscr{A}$ of $n$ complex lines in $C^{2}$ defined by a polynomial of the form $\prod_{l=1}^{n}\left(y-\alpha_{i}(x)\right)$.

\section{Labyrinth}

Let $\mathscr{A}$ be an arrangement of $n$ complex lines in $C^{2}$. Suppose that each line $H_{l} \in \mathscr{A}$ is defined by an equation $y=\alpha_{i}(x)$, where $\alpha_{i}$ is a linear function $\alpha_{i}: C \rightarrow$ C. Let $R_{i}(x)=\operatorname{Re}\left(\alpha_{i}(x)\right)$ and $I_{i}(x)=\operatorname{Im}\left(\alpha_{i}(x)\right)$. For any $1 \leq i<j \leq n$, the subset $L_{l, J}$ of the $x$-axis $C^{1}$, defined by

is a (real) line in $C^{1}$.

$$
L_{l, J}=\left\{x \in C^{1} ; R_{i}(x)=R_{j}(x)\right\},
$$

DEFINITION 3.1. We call the set

$$
\mathscr{L}(\mathscr{A})=\left\{L_{l, j} ; 1 \leq i<j \leq n\right\}
$$

the labyrinth of the arrangement $\mathscr{A}$.

Remark 3.2. (i) The notion of labyrinth was introduced by L. Rudolph [15] for any plane curve. Here we consider it in the arrangement context and call it by the name labyrinth. This notion was also used by Y. Orevkov in [12], where he called it the Rudolph's graph. 
(ii) For each line $L \in \mathscr{L}(\mathscr{A})$, there might be $i_{1}, \ldots, i_{k}$ with $1 \leq i_{1}<\cdots<$ $i_{k} \leq n$ such that

$$
L=\left\{x \in C^{1} ; R_{l_{s}}(x)=R_{l_{t}}(x), 1 \leq s<t \leq k\right\} .
$$

The number $k$ will be called the multiplicity of $L$. It is easy to see that after a suitable change of coordinates we can always assume that the multiplicity of any line $L$ in $\mathscr{L}(\mathscr{A})$ equals to 2 .

Clearly, each line $L_{l, J}$ divides the $x$-plane $C^{1}$ into two parts $L_{i, J}^{+}=\left\{x \in C^{1}\right.$; $\left.R_{i}(x)<R_{j}(x)\right\}$ and $L_{i, j}^{-}=\left\{x \in C^{1} ; R_{i}(x)>R_{j}(x)\right\}$. Each component of $C^{1} \backslash \mathscr{L}(\mathscr{A})$ can be then defined by $R_{s(1)}<\cdots<R_{s(n)}$ for a certain permutation $s$ of the set $\{1,2, \ldots, n\}$.

Similarly, for $1 \leq i<j \leq n$ we have the (real) line $L_{i, j}^{\prime}$ in $C^{1}$, defined by

$$
L_{i, j}^{\prime}=\left\{x \in C^{1} ; I_{i}(x)=I_{j}(x)\right\} .
$$

This line $L_{i, J}^{\prime}$ also divides $C^{1}$ into two parts $L_{i, j}^{\prime+}=\left\{x \in C^{1} ; I_{i}(x)<I_{j}(x)\right\}$ and $L_{i, J}^{\prime-}=\left\{x \in C^{1} ; I_{i}(x)>I_{j}(x)\right\}$. These lines $L_{i, J}^{\prime}$ will help to determine the braid $\theta\left(\Gamma_{l}\right)$ mentioned in the above section.

In the arrangement context, the points of $S(\mathscr{C})$ are usually called the multiple points of the arrangement $\mathscr{A}$. By definition, a multiple point $P$ of the arrangement $\mathscr{A}$ is the nonempty intersection of two or more hyperplanes of $\mathscr{A}$. The assumption on the genericity of the projection $p r_{1}$ implies that the multiple points of the arrangement $\mathscr{A}$ are distinct by their $x$-coordinates. In other words, the images of multiple points of $\mathscr{A}$ on the $x$-plane $C^{1}$ are pairwise distinct.

Let $x_{k} \in C^{1}$ be the image of a multiple point $P_{k}=\left(x_{k}, y_{k}\right)$ of $\mathscr{A}$ under the projection $p r_{1}$. Suppose that $P_{k}=\bigcap_{j=1}^{r} H_{l}$. Then it is clear that $x_{k}$ belongs to the lines $L_{l_{s}, l_{t}}, 1 \leq s<t \leq r$ of the labyrinth $\mathscr{L}(\mathscr{A})$. However, there might be another line $L \in \mathscr{L}(\mathscr{A})$, which does not belong to $\left\{L_{t_{s}, l_{t}} ; 1 \leq s<t \leq r\right\}$, going through this point $x_{k}$.

Definition 3.3. (i) The labyrinth $\mathscr{L}(\mathscr{A})$ is said to be good with respect to the multiple point $P_{k}=\bigcap_{J=1}^{r} H_{l_{J}}$ if there is not any line of $\mathscr{L}(\mathscr{A})$ except $L_{t_{s}, l_{t}}$; $1 \leq s<t \leq r$, going through $x_{k}$.

(ii) The labyrinth $\mathscr{L}(\mathscr{A})$ of an arrangement $\mathscr{A}$ is said to be proper if any line of $\mathscr{L}(\mathscr{A})$ has multiplicity 2 and it is good with respect to all multiple points of $\mathscr{A}$.

Remark 3.4. After a suitable change of coordinates we can assume that the labyrinth $\mathscr{L}(\mathscr{A})$ is good with respect to all multiple points of $\mathscr{A}$. So, from now on we always assume that the labyrinth $\mathscr{L}(\mathscr{A})$ of an arrangement $\mathscr{A}$ is proper.

\section{The braid monodromy of complex arrangement}

In this section we will determine the braid monodromy of an arrangement $\mathscr{A}=\left\{H_{i} ; i=1, \ldots, n\right\}$ of complex lines in $C^{2}$. 
Suppose that $f(x, y)=\prod_{l=1}^{n}\left(y-\alpha_{i}(x)\right)$ is the defining polynomial for $\mathscr{A}$. Let $\mathscr{P}=\left\{P_{1}, \ldots, P_{N}\right\}$ denote the set of multiple points of $\mathscr{A}$. These multiple points $P_{l}=\left(x_{l}, y_{i}\right)$ are the only singularities of $\mathscr{A}$. Remind that we always assume that the projection $p r_{1}: C^{2} \rightarrow C^{1}$ is generic with respect to the arrangement $\mathscr{A}$ and the labyrinth $\mathscr{L}(\mathscr{A})$ is proper. As indicated in the section $\S 1$, in order to determine the braid monodromy of $\mathscr{A}$ we have to determine all $\theta\left(\gamma_{i}\right)$ for a given good ordered system of generators $\left\{\gamma_{1}, \ldots, \gamma_{N}\right\}$ of $\pi_{1}\left(\boldsymbol{C}^{1} \backslash D(\mathscr{C})\right)$. And these $\theta\left(\gamma_{i}\right)$ 's are determined by local braid monodromies at $x_{l}$ and the braid homeomorphisms defined by moving the fiber of $p r_{1}$ along a chosen system of paths $\Gamma_{l}$. In [1] and [3], these datas was recorded by using the admissible 2graph or its generalization, the braided wiring diagram, respectively. Here we will read these datas from the labyrinth $\mathscr{L}(\mathscr{A})$ of the arrangement $\mathscr{A}$.

The intersection of $C_{x_{0}}=\left\{(x, y) \in C^{2} ; x=x_{0}\right\}$, the fiber of $p r_{1}$ over the base point $x_{0}$, with lines of $\mathscr{A}, C_{x_{0}} \cap\left(\bigcup_{l=1}^{n} H_{l}\right)$, consists of $n$ distinct points. When we move the fiber along a path in $x$-axis $C^{1}$, these points form a braid on $n$ strings. We will call the string corresponding to the hyperplane $H_{l}$ the $i^{\text {th }}$ string. In general these points have distinct real parts. A braiding will occur when the path intersects a line of the labyrinth $\mathscr{L}(\mathscr{A})$. In order to express $\theta\left(\gamma_{i}\right)$ in terms of the braid generator we need to recall of braids and braid groups.

A braid on $n$ strings can be viewed as the graph of the motion of $n$ points on a complex line from time $t=0$ to time $t=1$, satisfying

(i) These points remain distinct throughout the motion.

(ii) The sets of points at $t=0$ and $t=1$ are equal. There is a natural way to compose braids and to take the inverse of a braid. The isotopy classes of braids on $n$ strings form a group, $B(n)$, called the braid group. It has a presentation with generators $\sigma_{1}, \ldots, \sigma_{n-1}$ and defining relations

$$
\begin{aligned}
& \sigma_{l}, \sigma_{J}=\sigma_{J}, \sigma_{i} \text { if }|i-j| \geq 2 \\
& \sigma_{l}, \sigma_{l+1}, \sigma_{l}=\sigma_{i+1}, \sigma_{l}, \sigma_{i+1}
\end{aligned}
$$

where the generator $\sigma_{l}$ is illustrated in Figure 1 .

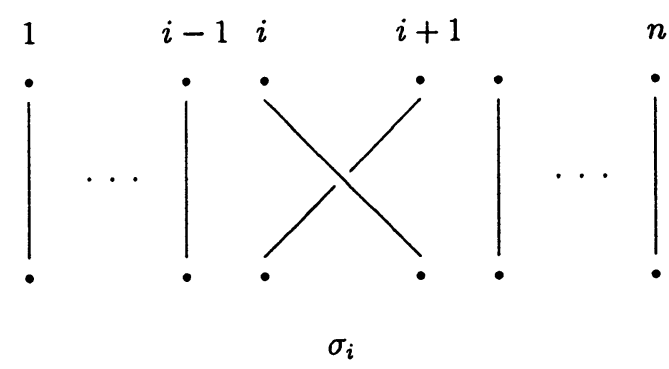

Figure 1 
For a subset $I=\{s+1, \ldots, s+r\}$ of $\{1, \ldots, n\}$, we call the "half-twist" on $I$ (see [2]) the following braid

$$
\Delta_{I}=\left(\sigma_{s+1} \sigma_{s+2} \cdots \sigma_{s+r-1}\right) \cdot\left(\sigma_{s+1} \sigma_{s+2} \sigma_{s+r-2}\right) \cdots\left(\sigma_{s+1} \sigma_{s+2}\right) \cdot \sigma_{s+1} .
$$

Geometrically, the half-twist $\Delta_{I}$ can be accomplished by holding the top of $j^{\text {th }}$ strings fixed, $s+1 \leq j \leq s+r$, and attaching the bottom of these strings to a rot and then turn it over once, while keeping fixed all other strings. See Figure 2.

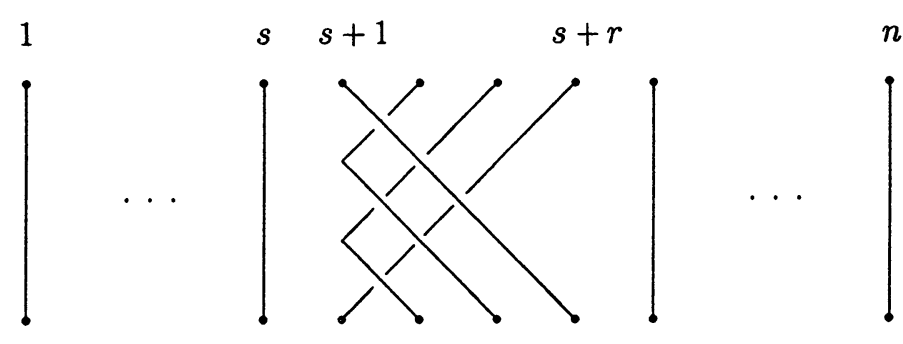

The half twist $\Delta_{I}$

Figure 2

The pure braid group, $P(n)$, is the kernel of the natural surjection $B(n) \rightarrow$ $S(n)$, where $S(n)$ denotes the symmetric group on $n$ letters. It is well known that $P(n)$ has a presentation with generators

$$
A_{\iota, J}=\sigma_{J-1} \sigma_{J-2} \cdots \sigma_{\imath+1} \sigma_{\imath}^{2} \sigma_{\imath+1}^{-1} \cdots \sigma_{J-1}^{-1},
$$

$1 \leq i<j \leq n$, and some certain defining relations.

Suppose that the base point $x_{0} \in \mathscr{C}^{1} \backslash D(\mathscr{C})$ is chosen in the component of $C^{1} \backslash \mathscr{L}(\mathscr{A})$, defined by $R_{1}<\cdots<R_{n}$. For a multiple point $P_{k}=\left(x_{k}, y_{k}\right)$, let $\mathscr{I}_{k}=\left\{i_{1}, \ldots, i_{r}\right\}$ be the set of all indices of those lines of $\mathscr{A}$ passing through $P_{k}$. It gives rise a partition of $\{1, \ldots, n\}$ as $\mathscr{L}_{k} \cup \mathscr{I}_{k} \cup \mathscr{U}_{k}$, where $\mathscr{L}_{k}=\left\{l_{1}, \ldots, l_{s}\right\}$, $\mathscr{I}_{k}=\left\{i_{1}, \ldots, i_{r}\right\}, \mathscr{U}_{k}=\left\{u_{1}, \ldots, u_{t}\right\}$, as follows. On the $x$-axis $C^{1}$, all components of $C^{1} \backslash \mathscr{L}(\mathscr{A})$ incident with $x_{k}$ must be of the following form

$$
R_{l_{1}}<\cdots<R_{l_{s}}<R_{l_{\sigma(1)}}<\cdots<R_{l_{\sigma(r)}}<R_{u_{1}}<\cdots<R_{u_{t}}
$$

where $\sigma$ is a certain permutation of $\{1, \ldots, r\}$. We call then the set $I_{k}=$ $\{s+1, \ldots, s+r\}$ the local index of the multiple point $P_{k}$.

LeMma 4.1. The local braid monodromy of $\mathscr{A}$ at $x_{k}$ is the full twist $A_{I_{k}}$ on the set $I_{k}$, where $A_{I_{k}}=\Delta_{I_{k}}^{2}$.

Proof. We consider the local situation at the multiple point $P_{k}$. Let $\mathscr{I}_{k}=$ $\left\{i_{1}, \ldots, i_{r}\right\}$ as above. Let choose the point $x_{k}^{\varepsilon}$ in the component of $C^{1} \backslash \mathscr{L}(\mathscr{A})$ defined by $R_{l_{1}}<\cdots<R_{l_{s}}<R_{l_{1}}<\cdots<R_{l_{r}}<R_{u_{1}}<\cdots<R_{u_{t}}$. For the sake of simplicity, the component $R_{l_{1}}<\cdots<R_{l_{s}}<R_{l_{\sigma(1)}}<\cdots<R_{l_{\sigma(r)}}<R_{u_{1}}<\cdots<R_{u_{t}}$ will be denoted simply by $R_{l_{\sigma(1)}}<\cdots<R_{l_{\sigma(r)}}$. 
First we consider the case $r=2$. It is illustrated in the Figure 3. We can choose $x_{k}^{\varepsilon} \in L_{i_{1}, l_{2}}^{\prime-}$. Then we move the fiber of $p r_{1}$ from $x_{k}^{\varepsilon}$ to a point in the component $R_{l_{2}}, R_{l_{1}}$ by a path lying in $L_{i_{1} l_{2}}^{\prime-}$. When the path crosses the line $L_{l_{1}, l_{2}}$ of the labyrinth a braid of $i_{1}^{t h}$ and $i_{2}^{t h}$ strings occurs. Because the cross point lies in $L_{i_{1}, l_{2}}^{\prime-}$, the $i_{1}^{\text {th }}$ string overcrosses the $i_{2}^{\text {th }}$ string at this cross point. Then if the local index of $P_{k}$ is $(i, i+1)$ we will get the braid $\sigma_{l}$. Now we continue to move the fiber of the projection $p r_{1}$ around $x_{k}$ and back to $x_{k}^{\varepsilon}$. Because the path, along which we move the fiber of $p r_{1}$, must go around $x_{k}$, when it crosses the line $L_{l_{1}, l_{2}}$ again the cross point must lie in $L_{i_{1}, l_{2}}^{\prime+}$. But this time we move from the component $R_{l_{2}}<R_{l_{1}}$ to the component $R_{l_{1}}<R_{l_{2}}$. So, once again we get the braid $\sigma_{l}$. It implies that the local braid monodromy at $x_{k}$ is $\sigma_{i}^{2}=A_{\{l, t+1\}}$.

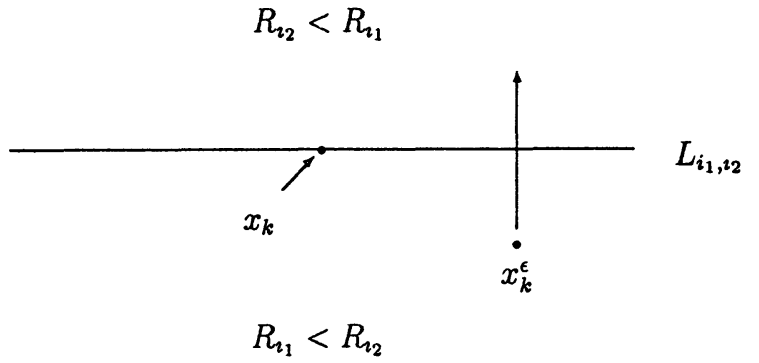

Figure 3

Next we consider the case $r>2$. As above let $I_{k}=\{s+1, \ldots, s+r\}$ be the local index of $P_{k}$. After a suitable isotopy, we may assume that locally at $x_{k}$, the point $x_{k}^{\varepsilon}$ is chosen in $L_{i_{1}, j}^{\prime-}, j \in\left\{i_{2}, \ldots, i_{r}\right\}$. It implies that when we move the fiber of $p r_{1}$ from $x_{k}^{\varepsilon}$ to a point in the opposite component of $C^{1} \backslash \mathscr{L}(\mathscr{A})$ at $x_{k}, R_{l_{r}}<\cdots<R_{l_{1}}$, the $i_{1}^{\text {th }}$ string will overcross all other $i_{j}^{\text {th }}$ strings, $2 \leq j \leq r$. An inductive argument shows that we obtain then the half twist $\Delta_{I_{k}}$ on the subset $I_{k}$. We continue to move the fiber of $p r_{1}$ around the point $x_{k}$ and back to $x_{k}^{\varepsilon}$. Similarly to the case $r=2$, here we obtain again the half twist $\Delta_{I_{k}}$. It proves that the local braid monodromy at $x_{k}$ is the full twist $A_{I_{k}}=\Delta_{I_{k}}^{2}$.

THEOREM 4.2. The braid monodromy of $\mathscr{A}$ is determined by

$$
\theta\left(\gamma_{k}\right)=\beta_{k} \cdot A_{I_{k}} \cdot \beta_{k}^{-1}
$$

$1 \leq k \leq N$, where $A_{I_{k}}$ is determined as in Lemma 4.1, $\beta_{k}$ is a braid which can be read off from the labyrinth $\mathscr{L}(\mathscr{A})$.

Proof. We do the global step in the usual way to construct the braid monodromy (cf. \$1). First we need to choose the paths $\Gamma_{k}$. For the multiple point $P_{k}=\left(x_{k}, y_{k}\right)$ let choose the point $x_{k}^{\varepsilon}$, near to $x_{k}$, as in Lemma 4.1, i.e. in the component of $C^{1} \backslash \mathscr{L}(\mathscr{A})$, given by

$$
R_{l_{1}}<\cdots<R_{l_{s}}<R_{l_{1}}<\cdots<R_{l_{r}}<R_{u_{1}}<\cdots<R_{u_{t}} .
$$


Then we take the path $\Gamma_{k}$ to be the minimal simple path, going from $x_{0}$ to $x_{k}^{\varepsilon}$. Now we will move the fiber of the projection $p r_{1}$ along this path $\Gamma_{k}$. Suppose that the path $\Gamma_{k}$ intersects the line $L_{l, j}$ of the labyrinth $\mathscr{L}(\mathscr{A})$. Then we will obtain a braiding of the $i^{\text {th }}$ string and $j^{\text {th }}$ string. To express this braiding in terms of braid generators suppose that locally at this intersection, $\Gamma_{k}$ goes from $R_{l_{1}}<\cdots<R_{l_{s}}<R_{l}<R_{\jmath}<R_{u_{1}}<\cdots<R_{u_{l}}$ to $R_{l_{1}}<\cdots<R_{l_{s}}<R_{\jmath}<R_{l}<R_{u_{1}}<\cdots<R_{u_{t}}$. Let call as above $\{s+1, s+2\}$ the local index of the intersection of $\Gamma_{k}$ and $L_{l, j}$. Then the braid of the $i^{t h}$ string and the $j^{\text {th }}$ string will be $\sigma_{s+1}$ if $\Gamma_{k}$ crosses $L_{l, J}$ at a point in the domain $L_{i, J}^{\prime-}$ and will be $\sigma_{s+1}^{-1}$ if $\Gamma_{k}$ crosses $L_{l, j}$ at a point in the domain $L_{i, j}^{\prime+}$. Recording successively all these braids when the fiber moves from $x_{0}$ to $x_{k}^{\varepsilon}$ we obtain the braid $\theta\left(\Gamma_{k}\right)$. Let $A_{I_{k}}$ is the full twist of the Lemma 4.1. Denoting the braid $\theta\left(\Gamma_{k}\right)$ by $\beta_{k}$ we have then the formula for the braid monodromy of $\mathscr{A}$

$$
\theta\left(\gamma_{k}\right)=\beta_{k} \cdot A_{I_{k}} \cdot \beta_{k}^{-1} .
$$

Note that we can use some formulas on the conjugation of $B(n)$ on $P(n)$ (see e.g. [2], [3]) to express the braid $\theta\left(\gamma_{k}\right)$ purely in term of pure braid generators. We will not repeat it here.

Remark 4.3. The use of the lines $L_{i, j}^{\prime}$ 's gives us a concrete method to determine which string is upper and which string is lower in the braiding of $i^{\text {th }}$ and $j^{t h}$ strings, i.e. to determine precisely all braids in the conjugation in $\theta\left(\gamma_{k}\right)$.

As noted in [11], the braid monodromy of $\mathscr{A}$ is closely related to the fundamental group of its complement $\pi_{1}\left(C^{l} \backslash \bigcup_{H \in \mathscr{A}} H\right)$. The intersection of the fiber $C_{x_{0}}$ of $p r_{1}$ over $x_{0}$ with hyperplanes of $\mathscr{A}$ consists of $n$ points. Then $C_{x_{0}} \backslash\left(C_{x_{0}} \cap\left(\bigcup_{H \in \mathscr{A}} H\right)\right)$ is a punctured complex line with $n$ removed points. Let $g_{1}, \ldots, g_{n}$ denote the generators of the free group $\pi_{1}\left(\boldsymbol{C}_{x_{0}} \backslash\left(\boldsymbol{C}_{x_{0}} \cap\left(\bigcup_{H \in \mathscr{A}} H\right)\right)\right)$. The braid group $B\left[\boldsymbol{C}_{x_{0}}, \boldsymbol{C}_{x_{0}} \cap\left(\bigcup_{H \in \mathscr{A}} H\right)\right]$ can be naturally considered as a group of automorphisms of $\pi_{1}\left(C^{l} \backslash \bigcup_{H \in \mathscr{A}} H\right)$. Let identify $g_{1}, \ldots, g_{n}$ with their images in $C^{l} \backslash \bigcup_{H \in \mathscr{A}} H$ by the homomorphism induced from the embedding $C_{x_{0}} \backslash\left(C_{x_{0}} \cap\right.$ $\left.\left(\bigcup_{H \in \mathscr{A}} H\right)\right) \subset C^{l} \backslash \bigcup_{H \in \mathscr{A}} H$. Remind that for each multiple point $P_{k}, 1 \leq k \leq N$ we denote by $\mathscr{I}_{k}$ the set of indices of all lines of $\mathscr{A}$ going through $P_{k}$. Then we have the following corollary (cf. [10]).

COROLlaRY 4.4. The fundamental group of the complement to the arrangement $\mathscr{A}, \pi_{1}\left(C^{l} \backslash \bigcup_{H \in \mathscr{A}} H\right)$, is generated by elements $g_{1}, \ldots, g_{n}$, with the defining relations

$$
g_{i}=\beta_{k} \cdot A_{L_{k}} \cdot \beta_{k}^{-1} \cdot g_{i}
$$

$i \in \mathscr{I}_{k}, k=1, \ldots, N$.

Remark 4.5. From the above presentation of $\pi_{1}\left(C^{l} \backslash \bigcup_{H \in \mathscr{A}} H\right)$ we can simplify the defining relations to get the presentation given in [6]. If instead of 
the system of $\Gamma_{k} ; k=1, \ldots, N$ we follow the way of Arvola to choose a PL graph in the $x$-plane $C^{1}$, by the method mentioned above using the labyrinth we can also obtain the Arvola's presentation for the fundamental group of the complement.

COROLlARY 4.6. The labyrinth of an arbitrary arrangement $\mathscr{A}$ in $C^{2}$ determines the homotopy type of its complement.

Proof. According to Libgober [10], the complement of an arrangement $\mathscr{A}$ is homotopy to the standard 2-complex modelled on the braid monodromy presentation of its $\pi_{1}$. The braid monodromy presentation of $\pi_{1}$ of its complement given in the Corollary 4.4 is determined by its labyrinth. So, the corollary is proved.

\section{REFERENCES}

[1] W. A. Arvola, The fundamental group of the complement of an arrangement of complex hyperplanes, Topology, 31 (1992), pp. 757-766.

[2] J. Birman, Braids Links and Mapping Class Groups, Ann. of Math. Stud., 82, Princeton Univ. Press, 1975.

[3] D. C. CohEn AND A. I. SUCIU, The braid monodromy of plane algebraic curves and hyperplanes arrangements, preprint 1996.

[4] R. CoRDOviL, Braid monodromy group of arrangements of hyperplanes, preprint 1994.

[5] NGuYen, V. D., The fundamental group the complement of complexified real arrangements, Ann. Sc1. Math. Québec, 18 (1994), pp. 157-167.

[6] NGUYen, V. D. AND HA, H. V., The fundamental group of complex hyperplane arrangement, Acta Math. Vietnam., 20 (1995), pp. 31-41.

[7] H. A. Hamm and Le Dung Trang, Un théorème de Zarıskı du type Lefschetz, Ann. Sc1. École Norm. Sup., 6 (1973), pp. 317-366.

[ 8 ] E. Hironaka, Abelian covering of the complex projective plane branched along configurations of real lines, Mem. Amer. Math. Soc., 105 (1993), no. 502.

[9] E. R. VAN KAMPEN, On the fundamental group of an algebraic curve, Amer. J. Math., 55 (1933), pp. 255-260.

[10] A. Libgober, On the homotopy type of the complement to plane curves, J. Reine Angew Math., 367 (1986), pp. 103-114.

[11] B. Morshezon, Stable branch curves and braid monodromies, Algebraic Geometry, Lecture Notes in Math., 862, Springer-Verlag, 1981, pp. 107-192.

[12] Y. Orevkov, Rudolph's diagrams and analytıcal realization of Vitushkın's coverıng, preprınt.

[13] P Orlik AND H. Terao, Arrangements of Hyperplanes, Grundlehren Math. Wiss., 300, Sprınger-Verlag, 1992.

[14] R. RANDELL, The fundamental group of the complement of a union of complex hyperplanes, Invent. Math., 69 (1982), pp. 103-108; Correction, Invent. Math., 80 (1984), pp. 467-468.

[15] L. RUdOlPH, Algebraic functions and closed braids, Topology, 22 (1983), pp. 191-202.

[16] M. Salvetti, Arrangements of lines and monodromy of plane curves, Compositio Math., 68 (1988), pp. 103-122. 
[17] O. ZARISKI, On the existence of algebraic function of two variables possesing a given branch curve, Amer. J. Math., 51 (1929), pp. 305-328.

SECTION OF TOPOLOGY-GEOMETRY

HaNOI Institute OF Mathematics

P.O. BOX 631, 10000 HANOI

VIETNAM

E-mail: vietdung@thevinh.ncst.ac.vn 